\title{
Metal $\beta$-Diketoiminate Precursor use in Aerosol Assisted Chemical Vapour Deposition of Gallium- and Aluminium-Doped Zinc Oxide
}

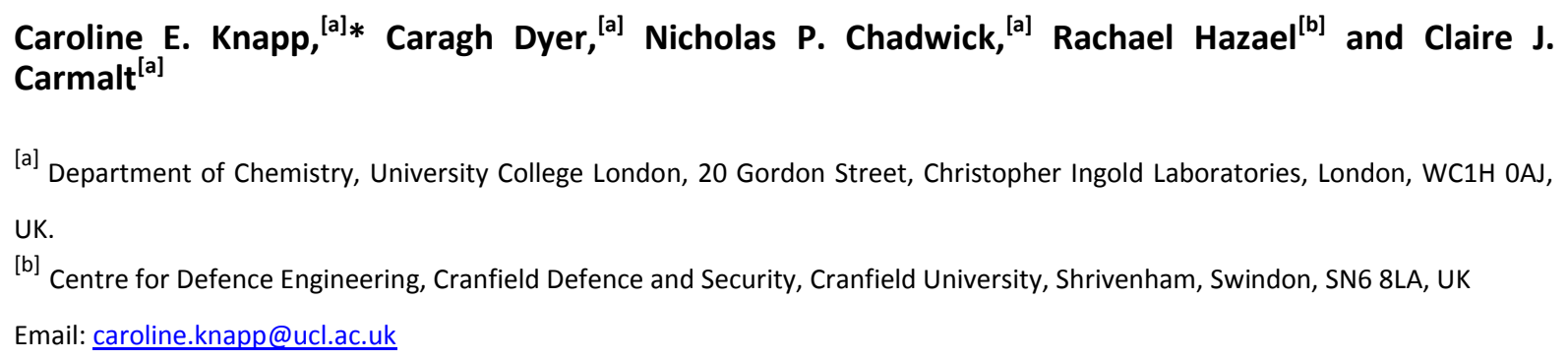

\section{Abstract}

Aerosol assisted chemical vapour deposition (AACVD) has been used to deposit thin films of $\mathrm{ZnO}$ from the single-source precursor $\left[\mathrm{Zn}\left(\left(\mathrm{OC}(\mathrm{Me}) \mathrm{CHC}(\mathrm{Me}) \mathrm{N}\left({ }^{i} \mathrm{Pr}\right)\right)_{2}\right]\right.$ (1) affording highly transparent ( $>80 \%$ ) and conductive films (sheet resistance $~ 70$ $\mathrm{K} \Omega / \mathrm{sq}$ ). Extension of this AACVD method whereby related precursors of the type, $\left[\mathrm{R}_{2} \mathrm{M}(\mathrm{OC}(\mathrm{Me}) \mathrm{CHC}(\mathrm{Me}) \mathrm{N}(\mathrm{Pr})](\mathrm{R}=\mathrm{Et}, \mathrm{M}=\mathrm{Al}(\mathbf{2}) ; \mathrm{R}=\mathrm{Me}, \mathrm{M}=\mathrm{Ga}(\mathbf{3}))\right.$, isolated as oils, were added to the precursor solution allowed for the deposition of aluminium- and gallium-doped ZnO (AZO and GZO) films, respectively. Complexes 1-3 were characterised by elemental analysis, NMR and mass spectrometry. Films were deposited in under 30 minutes at $400{ }^{\circ} \mathrm{C}$, from $\mathrm{CH}_{2} \mathrm{Cl}_{2}$ /toluene solutions with a $\mathrm{N}_{2}$ carrier gas. Herein we report the bulk resistivity, $\rho$, of AZO $(0.252 \Omega \mathrm{cm})$ and GZO $(0.756 \Omega \mathrm{cm})$ films deposited from this novel approach. All the films transparency exceeded $80 \%$ in the visible, $X$-ray diffraction (XRD) showed all films to crystallise in the wurzite phase whilst X-ray photoemission spectroscopy (XPS) confirmed the presence of the Al and Ga dopants in the films, and highlighted the low C-contamination $(<5 \%)$ this route offers. Investigation of a mechanism analogous to the Kirkendall effect confirmed that heating of GZO films at $1000^{\circ} \mathrm{C}$ produced the spinel structure $\mathrm{GaZn}_{2} \mathrm{O}_{4}$.

\section{Keywords: Zinc oxide, thin films, Al doping, Ga doping, AACVD}

\section{Introduction}

Metal oxide thin films have garnered a large amount of attention in the scientific community due to their wide range of existing and potential applications, particularly their use in transparent conducting oxide (TCO) coatings.[1] TCOs are optically transparent and electrically conductive making them the ideal candidates for optoelectronic and photovoltaic applications. Currently, the fastest growing use of TCOs is in flat panel display technology, an area that covers computer and smartphone screens for consumer electronics but also for the military and medical industries.[2] There is also great demand for their use in the glass industry particularly in electrochromic windows. TCOs often comprise of $n$-doped tin, zinc or indium oxides. As a 
general rule, a TCO should possess a transmittance of $>80 \%$, a carrier concentration of $10-20 \mathrm{~cm}^{-3}$ and the host matrix must comprise of a wide band gap metal oxide ( $>3 \mathrm{eV}$ ) and be doped until rendered degenerate to exhibit metallic-like electrical conductivity.[3] However, these desirable properties can conflict as the large band gap required for optical transparency is detrimental to the low resistivity required $\left(\sim 10^{-4} \Omega \mathrm{cm}\right)$, often causing problems for the doping process. Indium tin oxide (ITO) films are the industrial standard with resistivites of $\sim 1 \times 10^{-4} \Omega \mathrm{cm}$, a large bandgap of $4 \mathrm{eV}$ and transparency in the majority of the visible region. Indeed, the optical and electrical properties are difficult to match, however alternatives to ITO are widely sought with a view to lowering the cost and simplifying deposition techniques.

A range of doped zinc oxides are a viable alternative to ITO.[4] The large bandgap, ease of doping and high conductivity of zinc oxide is consistent with the prerequisites for TCOs however doping is necessary due to the low carrier concentration and thus high resistivity of pure $\mathrm{ZnO}$.[5] The doping of zinc oxide occurs through $n$-type doping where impurities are introduced into the crystal lattice to cause an excess of negative charge carriers. Doped zinc oxides have been widely explored with dopants, such as arsenic,[6] indium[7] and manganese.[8] Arguably, the most notable dopant is aluminium, forming Al-doped ZnO (AZO) which is currently used in the photovoltaic industry, with reported resistivities as low as $2.4 \times 10^{-4} \Omega \mathrm{cm}$ and a large band gap of 3.4-3.9 eV.[9] Furthermore, although the properties of ITO are considered superior, the low cost, availability and low toxicity of zinc and aluminium are appealing industrially. It was reported that AZO exhibits a high stability at temperatures $>700 \mathrm{~K}$ and are the only films stable in a gaseous plasma containing hydrogen[10] also more recently it has been reported that combining both aluminium and gallium as dopants offers a balance between chemical stability and conductivity.[11] Various doping concentrations have been explored in the literature with most lying between 2-10 at.\%. Muiva et al. reported the optimum doping concentration to be 2 at.\% for enhanced conductivities and transmittance $>85 \%$.[12]

Group III elements are common dopants for $\mathrm{ZnO}[13]$ and moving down the group from Al to Ga as a dopant gives Ga-doped zinc oxide (GZO), another alternative to ITO. Studies suggest that gallium doping is more effective than aluminium doping as the lower reactivity of gallium allows it to be easily controlled during the doping process. Additionally, the smaller Ga-O bond length minimises the deformity of the $\mathrm{ZnO}$ crystal lattice allowing higher concentrations of the dopant to be introduced into the host matrix. Furthermore, with a reported resistivity between $4-5 \times 10^{-4} \Omega \mathrm{cm}$ it also offers similarly impressive electrical properties.[14,15] In addition to dopant concentration, electrical properties of films can also depend on film thickness. Fortunato et al. discovered that increasing the GZO film thickness also increased the electron mobility as defects are more common in thinner films thus scattering charge carriers. However, saturation of the resistivity occurred at thicknesses that exceeded $500 \mathrm{~nm}$.[15]

Recently studies of films of AZO and GZO deposited via AACVD showed the correlation between dopant levels and resultant resistivity.[16,17] Commercially procured zinc acetylacetonate, aluminium chloride and gallium chloride were used to synthesize $\mathrm{ZnO}$ doped with 5, 10, 15 and 20 at.\% Al or Ga. The resultant films showed 
transparency greater than $80 \%$ and resistivities in the order of $10^{-3} \Omega \mathrm{cm}$.[18] Earlier this year both Al (1.5 at.\%) and Ga (1.5 at.\%) doped ZnO powders and thin films (produced via AACVD) were also reported with resistivity values of $5.6 \times 10^{-4} \Omega \mathrm{cm}$ and $5.7 \times 10^{-3} \Omega \mathrm{cm}$, respectively using a microwave assisted synthesis.[19] In both of these reports, where commercially available precursors were used, carbon and/or chlorine contamination was reported in the resultant materials.

Research into metal oxide thin film synthesis has been carried out hand in hand with the development of metal-organic precursors, usually metal alkoxides or $\beta$-diketonates designed to limit contamination.[20] These are single-source precursors containing a direct metal-oxygen bond that can either be synthesised or in some cases are commercially available. $\beta$-ketoimine ligands can form a complex with a metal centre, forming a delocalised ring including $\mathrm{O}$ and $\mathrm{N}$ donor atoms. The use of the ligand has been reported to increase thermal stability and lower the melting point. The move from $\beta$-diketonates to $\beta$-dikeiminate facilitates the incorporation of a $\mathrm{N}$ atom into the delocalised ring which can be further functionalised - allowing various properties of the precursors to be tuned with the careful selection of R group on the N.[21] It has been shown recently that varying the $R$ group on the nitrogen atom in a range of $\beta$-diketoiminate ligands dramatically altered the properties of the resultant aluminium and gallium containing precursors.[22]

The ability to isolate a precursor as an oil, can be advantageous, particularly for use in AACVD, where precursors must be soluble in order to generate the aerosol mist.[9] Ligand modification is not limited to functionalisation on the nitrogen atom, it can include the fluorination of the alkyl backbone; these electron withdrawing groups can reduce the strength of the intermolecular forces via increased electron density at the metal centre resulting in a more volatile product. However contamination and the risk of metal corrosion from fluoride species must be taken into consideration. $[3,20] \mathrm{A}$ range of zinc $\beta$-diketoiminate compounds have been successfully utilized in the CVD of ZnO films, $[23,24]$ and it is proposed that an extension to this groundwork could include the incorporation of Al and Ga dopants to improve the conductivity of the resultant materials.

Previous reports of AZO and GZO synthesis describe chlorine contamination as a result of precursor ligand design, in this paper, to circumnavigate this we combine the use of an identical $\beta$-diketoiminate ligand motif on zinc, aluminium and gallium compounds with AACVD in order to facilitate doping into the $\mathrm{ZnO}$ lattice. The use of the solution based technique, AACVD, overcomes the need for volatile precursors whilst the metal $\beta$ diketoiminate precursors presented herein exhibit similar decomposition mechanisms. The precursors therefore decompose cleanly, readily dope the ZnO framework (at $400{ }^{\circ} \mathrm{C}$ ) and produce increasingly conductive thin films of $\mathrm{ZnO}, \mathrm{GZO}$ and $\mathrm{AZO}$, respectively, free from chlorine contamination. 
$\mathrm{ZnEt}_{2}, \mathrm{AlEt}_{3}$ and $\mathrm{GaMe}_{3}$ are pyrophoric substances that can ignite spontaneously in air and react violently with water. Therefore, all reactions involving air sensitive materials were carried out under a dry dinitrogen (99.99\% from BOC) atmosphere using an MBraun glove box and Schlenk techniques. All experimental should be conducted in a fume hood. Following the deposition films were air and moisture stable and were safe to handle as any reactive species leave via the reactor exhaust during the AACVD process. All solvents used were stored in alumina columns and dried, such that the water concentration was $5-10 \mathrm{ppm}$. $\mathrm{AlEt}_{3}$ and $\mathrm{GaMe}_{3}$ were procured from SAFC Hitech, $\mathrm{ZnEt}_{2}$ and all other chemicals were purchased from Aldrich and stored appropriately.

${ }^{1} \mathrm{H}$ and ${ }^{13} \mathrm{C}$ NMR spectra were obtained on a Bruker AMX500 spectrometer, operating at $500.13 \mathrm{MHz}$, using $\mathrm{CD}_{2} \mathrm{Cl}_{2}$ or $\mathrm{CDCl}_{3}$ which were dried and degassed over molecular sieves prior to use; ${ }^{1} \mathrm{H}$ and ${ }^{13} \mathrm{C}$ chemical shifts are reported relative to $\mathrm{SiMe}_{4}(\delta$ 0.00). IR spectra were recorded using a Shimadzu FTIR-8200 spectrometer, operating in the region of $4000-400 \mathrm{~cm}^{-1}$. Mass spectra were obtained using a Micromass 70-SE spectrometer using Chemical Ionoisation ( $\mathrm{Cl}$ ) with methane reagent gas. Elemental Analysis was carried using Elemental Analyser (CE-440) (Exeter Analytical Inc). The instrument used for thermal analysis was a Netzsch Jupiter. All measurements were carried out with the precursor sample sealed in an aluminium pan. The data was recorded from room temperature to $600^{\circ} \mathrm{C}$ (see S.I.).

Synthesis of the protonated ligand: $[\mathrm{OC}(\mathrm{Me}) \mathrm{CHC}(\mathrm{Me}) \mathrm{NH}(\mathrm{Pr}))]$, [Zn $\left(\mathrm{OC}(\mathrm{Me}) \mathrm{CHC}(\mathrm{Me}) \mathrm{N}\left(\mathrm{Pr}^{\prime}\right)_{2}\right]$ (1) and $\left[\mathrm{Et}_{2} \mathrm{Al}\left(\mathrm{OC}(\mathrm{Me}) \mathrm{CHC}(\mathrm{Me}) \mathrm{N}\left({ }^{\prime} \mathrm{Pr}\right)\right)\right](2)$ are consistent with literature and are included in $\mathbf{S . 3 .}$

The $\beta$-ketoiminate ligand, $\left.\left[\mathrm{OC}(\mathrm{Me}) \mathrm{CHC}(\mathrm{Me}) \mathrm{NH}\left({ }^{(} \mathrm{Pr}\right)\right)\right](1.00 \mathrm{~g}, 7.0 \mathrm{mmol})$ in toluene $(20 \mathrm{~mL})$ was added dropwise to a solution of $\mathrm{GaMe}_{3}(0.81 \mathrm{~g}, 7.0 \mathrm{mmol})$ in toluene $(20 \mathrm{~mL})$ at $-78^{\circ} \mathrm{C}$. This was stirred overnight and the solvent was removed in vacuo to yield a viscous yellow oily product $1(70 \%) .{ }^{1} \mathrm{H} N M R \delta / p p m\left(C_{6} \mathrm{D}_{6}\right)$ : $4.56(\mathrm{~s}, 1 \mathrm{H}, \mathrm{COCH}), 3.21\left(\mathrm{~m}, 1 \mathrm{H}, \mathrm{CH}(\mathrm{Me})_{2}\right), 1.66(\mathrm{~s}, 3 \mathrm{H}, \mathrm{MeCO}), 1.34(\mathrm{~s}, 3 \mathrm{H}, \mathrm{CNMe}), 1.10\left(6 \mathrm{H}, \mathrm{d}, \mathrm{NCH}(\mathrm{Me})_{2}\right)$, 0.14 (4H, m, GaMe). ${ }^{13} \mathrm{C}\left\{{ }^{1} \mathrm{H}\right\}$ NMR $\delta / \mathrm{ppm} 180.0$ (CO), 165.2 (CN), 82.3 (CH), 39.7 (NC(Me) $)$ ), 26.2 (OCMe), 23.5 ( $\left.\mathrm{NC}(\mathrm{Me})_{2}\right), 22.2$ (COMe), 1.8 (GaMe). M/S: m/z [ES] + 239.99. Elemental anal. calc. \%: C: 50.05, H: 8.40, N: 5.84, found \%: C: 51.3, H: 9.1, N: 5.99 .

\subsection{Chemical vapour deposition}

AACVD reactions took place within a fume hood. Depositions were carried out on $\mathrm{SiO}_{2}$ coated barrier glass substrates with proportions: $90 \mathrm{~mm} \times 45 \mathrm{~mm} \times 4 \mathrm{~mm}$ within a cold walled, horizontal bed reactor. Two glass substrates were used to form a bottom plate which sat directly on top of the graphite heating block and a top plate positioned above it with a $0.5 \mathrm{~cm}$ gap between them. The desired thin film was deposited on the bottom plate, whilst the top plate reduces turbulence, ensuring laminar gas flow. The glass substrates were cleaned thoroughly using water, ${ }^{i} \mathrm{PrOH}$ and acetone and then dried in air before each use. The temperature of 
under a flow of the $\mathrm{N}_{2}$ carrier gas and the aerosol entering through the brass baffle. The reactor was sealed

142 with a cylindrical quartz tube capped by two stainless steel end plates with an exhaust. Before the aerosol

143 was formed, the precursor was weighed out into a glass bubbler and appropriate solvent added. Once the

144 reactor had reached the required temperature, the glass bubbler was attached to the reactor using PTFE

145 tubing via a one way glass T-piece. The flow meter, controlling the $\mathrm{N}_{2}$ carrier gas flow, was diverted through

146 the T-piece diverting the aerosol mist generated by a humidifier into the reactor via the baffle. To create the

147 aerosol, an ultrasonic humidifier containing a piezoelectric device, which functions at $20 \mathrm{kHz}$ was used. XRD

148 was carried out with a Bruker D8 Discover X-ray diffractometer using monochromatic $\mathrm{Cu} K_{\alpha 1}$ and $\mathrm{Cu} K_{\alpha 2}$

149 radiation of wavelengths 1.54056 and $1.54439 \AA$ respectively, emitted in an intensity ratio of 2:1, voltage $=40$

$150 \mathrm{kV}$; current $=40 \mathrm{~mA}$. SEM was performed using a Philips XL30 FEG with an electron beam accelerating energy

151 of $30 \mathrm{kV}$. XPS profiling was performed using a Thermo Scientific K-Alpha XPS system using monochromatic Al

$152 K_{\alpha}$ radiation at $1486.6 \mathrm{eV} X$-ray source. CasaXPS software was used to analyse the data with binding energies

153 referenced to an adventitious C 1s peak at $284.8 \mathrm{eV}$. UV/Vis/NIR transmission spectra were recorded using a

154 PerkinElmer Lambda 950 spectrometer in the range of $300-1400 \mathrm{~nm}$ with an air background.

155

156

157

158

159

160

161

162

163

164

165

166

167

168

169

170

171

172

173

174

\subsection{General procedures}

Due to the air sensitive nature of the precursors, standard Schlenk and glovebox (MBraun Unilab) techniques were employed and all precursors and solvents were kept under a dry $\mathrm{N}_{2}$ atmosphere. Solvents were added to the vessel containing the precursor using an oven-dried cannula and then attached to the AACVD apparatus under a flow of $\mathrm{N}_{2}$. A number of different flow rates were tested ranging between $1-2 \mathrm{~L} / \mathrm{min}$ as well as a variety of different deposition temperatures. The AACVD reaction lasted between 30-60 min and the reactor was set to cool to room temperature, still under a flow of $\mathrm{N}_{2}$. Many attempts were made to optimise the AACVD reaction of the precursor testing different solvents, flow rates, temperatures and masses of precursor.

\subsection{AACVD of $Z n O$ from 1}

1 (0.2 g, $0.578 \mathrm{mmol}$ ) was added to a glass flask in the glove box. A small amount of $\mathrm{CH}_{2} \mathrm{Cl}_{2}$ (ca. $10 \mathrm{~mL}$ ) was added to dissolve the precursor followed by toluene $(c a .30 \mathrm{~mL})$. The resultant solution was a transparent, pale yellow/orange colour. The AACVD reactor was heated to $400{ }^{\circ} \mathrm{C}$ whilst under a flow of $\mathrm{N}_{2}$ gas at a flow rate of $1 \mathrm{~L} / \mathrm{min}$. The flow of $\mathrm{N}_{2}$ was then diverted into the glass bubbler through the precursor solution allowing the aerosol mist to be directed into the AACVD reactor. The deposition took 30 min and the reactor was cooled to room temperature under a flow of $\mathrm{N}_{2}$ gas before the substrate was removed.

\subsection{AACVD of $A Z O, G Z O$ and $\mathrm{GaZn}_{2} \mathrm{O}_{4}$.}

The experimental procedure described above was repeated several times, films of AZO from one-pot of 1 and $\mathbf{2}$ in a 1:0.05 ratio $(0.5 \mathrm{mmol}$ scale). GZO films were deposited as above using compounds $\mathbf{1}$ and $\mathbf{3}$ (also onepot) in a ratio of 1:0.05 (0.5 mmol scale). The experimental procedure described for GZO was repeated with 
the addition of a piece of glass quartz $(2 \mathrm{~cm} \times 2 \mathrm{~cm})$ placed on top of the substrate. The quartz was then transferred to a Carbolite cwf $13 / 13$ furnace and annealed at $1000{ }^{\circ} \mathrm{C}$ overnight, in order to carry out XRD analysis.

\section{Results and discussion}

\subsection{Synthesis of 1-3}

$\beta$-ketoimine compounds, formed in a simple condensation reaction have been widely explored as ligands for a range of metals and used in the synthesis of successful precursors for metal oxide thin films.[22,23] Following the synthesis and isolation of the ligand, $[(\mathrm{Me}) \mathrm{CN}(\mathrm{H})\{\mathrm{Pr}\}-\mathrm{CHC}(\mathrm{Me})=0]$, two equivalents were reacted with diethyl zinc in toluene and a white precipitate of $\mathbf{1}$ was obtained and isolated in good yield, after being filtered and washed with hexane. Conversely compounds $\mathbf{2}$ and $\mathbf{3}$ were not isolated as precipitates after an overnight reflux of a 1:1 reaction of the aforementioned ligand with $\mathrm{AlEt}_{3}$ and $\mathrm{GaMe}_{3}$ which yielded oils of $\mathbf{2}$ and $\mathbf{3}$, respectively. Formation of $\mathbf{1}$ and $\mathbf{2}$ were confirmed via $^{1} \mathrm{H},{ }^{13} \mathrm{C}, \mathrm{MS}$ and $\mathrm{EA}$ and were consistent with literature.[22,23] The proton resonances in the NMR of $\mathbf{3}$ for the Ga-ligand environments were significantly shifted from ${ }^{1} \mathrm{H}$ environments observed for the protonated ligand: [(Me) $\left.\mathrm{CN}(\mathrm{H})\left\{{ }^{i} \mathrm{Pr}\right\}-\mathrm{CHC}(\mathrm{Me})=\mathrm{O}\right]$ alone, evidencing the formation of $\mathbf{3}$. Elemental analysis and mass spectrometry confirm the isolation of $\mathbf{3}$, futhermore, the 2:1 ratio of methyl group proton environments to the Ga-ligand proton environments in the ${ }^{1} \mathrm{H}$ NMR support the formation of $\mathbf{3}$ (Scheme 1 ).

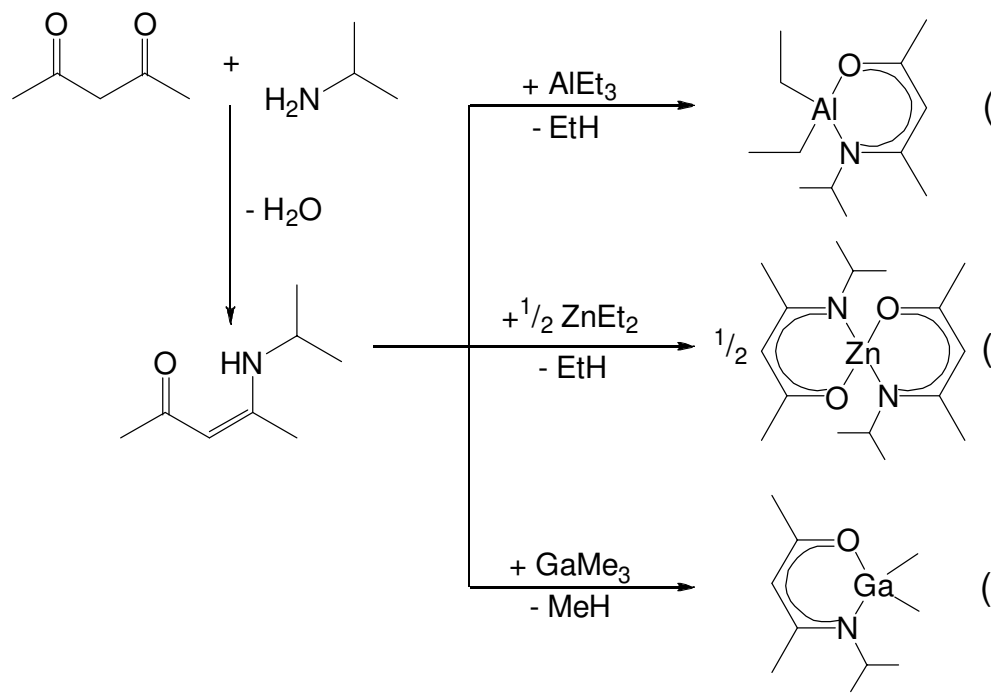

(2)

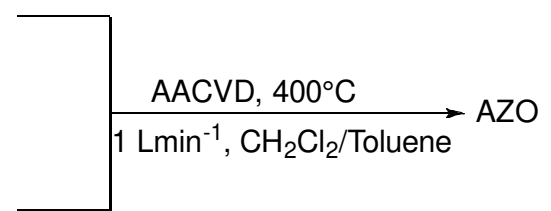

(1)

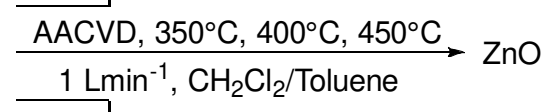

(3) AACVD, $400^{\circ} \mathrm{C} \rightarrow \mathrm{GZO}$ $1 \mathrm{Lmin}^{-1}, \mathrm{CH}_{2} \mathrm{Cl}_{2} /$ Toluene

Scheme 1. Synthesis of $\beta$-ketoiminate metal complexes 1-3 and the conditions for the AACVD of thin films of $\mathrm{ZnO}, \mathrm{AZO}$ and GZO.

\section{2. $A A C V D$ of $Z n O, A Z O$ and $G Z O$}

Following optimisation of the AACVD process (see S.I.) compound $\mathbf{1}$ was used to deposit thin films of $\mathrm{ZnO}$ at $400{ }^{\circ} \mathrm{C}$. Films of AZO were achieved at $400{ }^{\circ} \mathrm{C}$ via a one-pot AACVD charged with $\mathbf{1}$ and $\mathbf{2}$ in a 1:0.05 ratio. Following the procedure for the synthesis of thin films of $\mathrm{AZO}$ (at $400{ }^{\circ} \mathrm{C}$ ), GZO films were also successfully 

utilised (see S.I.). The films of ZnO, AZO and GZO were transparent displaying a slight brown tinge, indicative of carbon contamination. All films were adherent to the glass substrate after wiping, scratching with a scalpel, the scotch tape test and treating with acetone and other organic solvents. The films perished when left in nitric acid overnight.

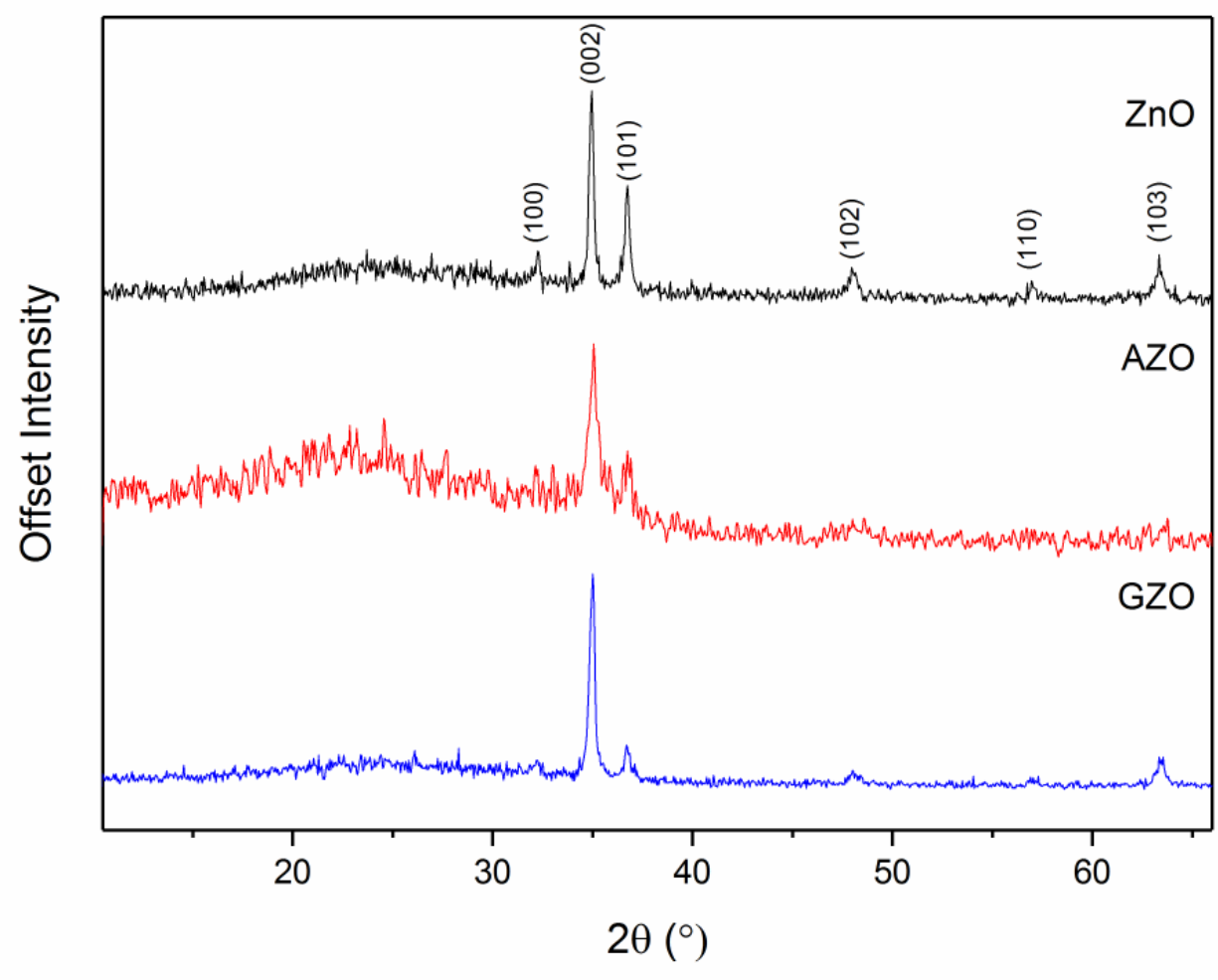

Fig. 1: XRD pattern showing: ZnO deposited by AACVD of 1 at $400^{\circ} \mathrm{C}$ (black);[10] AZO deposited from 1 and 2 (red); GZO deposited from $\mathbf{1}$ and $\mathbf{3}$ (blue). Peaks verify the hexagonal wurzite structure expected.

The XRD pattern for films deposited from 1 show alignment to peaks typical of zinc oxide, with preferential orientation for the (002) peak indicating orientation along the $c$ axis.[25] Using the Le Bail method in GSAS, the lattice parameters of the ZnO films were determined to be $a=3.2367(5) \AA$ and $b=5.1613(5) \AA$.

AZO films deposited from a one-pot mixture of $\mathbf{1}$ and $\mathbf{2}$ show a significant decrease in peak intensity with $\mathrm{Al}$ doping in their XRD pattern. This may be due to the varying film thickness which is well known to affect the intensity of the XRD peaks. A shift in the peak position when doping occurs due to the change in lattice parameters that accompanies the introduction of dopant atom into the host matrix, shifting was observed when compared to the $2 \theta$ values of the undoped zinc oxide films. Firstly for the (002) peak from $34.85^{\circ}$ to $35.00^{\circ} 2 \theta$ and the (100) peak from $31.40^{\circ}$ to $32.15^{\circ} 2 \theta$. The replacement of $\mathrm{Zn}^{2+}$ ions with the smaller $\mathrm{Al}^{3+}$ ions 
217 films to investigate the effect of doping on the crystal structure. Using the aforementioned methods the

218 lattice parameters were determined to be $a=3.2353(5) \AA$ and $b=5.1785(5) \AA$.

219 In the XRD patterns of films of GZO deposited from one-pot mixtures of $\mathbf{1}$ and $\mathbf{3}$ preferential orientation 220 remained for the (002) peak, and the intensity of the peaks was decreased in comparison with undoped $\mathrm{ZnO}$.

221 A very slight shift in the (002) peak was observed in the spectrum from $34.85^{\circ}$ in the undoped $\mathrm{ZnO}$ film to

$22234.9^{\circ} 2 \theta$ in the GZO. However, the difference in size between the ionic radii of zinc and gallium is relatively 223 small, therefore shifting may not occur as the distortion of the crystal lattice could be minimal, with similar 224 observations have been reported in the literature.[14] The shift is smaller than that observed for the AZO 225 films which is expected due to the smaller difference in ionic radii between zinc and gallium compared to that 226 of zinc and aluminium.

227 As with AZO, lattice parameters of the unit cell were calculated for GZO films to investigate the effect of 228 doping on the crystal structure. Using the Le Bail method lattice parameters were determined to be $229 a=3.2257(5) \AA$ and $b=5.1496(5) \AA$. . The data reveals a slight shift in the parameters showing a small effect on 230 the crystal structure by the dopant atoms. In the case of gallium, it is well reported in the literature that 231 incorporation into the $\mathrm{ZnO}$ crystal structure causes the (002) to shift to a higher angle side, reflected in the 232 decrease in the c parameter from $5.16 \AA$ to $5.15 \AA$.[27]

233 Energy dispersive X-ray (EDX) analysis of films of ZnO deposited from 1 confirmed the presence of zinc but 234 could not be used to give a metal to oxygen ratio due to breakthrough to the substrate. Carbon contamination was apparent from the EDX data ( 15 at.\% carbon). The EDX analysis of the AZO films confirmed the Al content of the films to be 4.8 at.\%, closely corroborating the experimental concentrations used. Films of GZO deposited from 1 and 3 were analysed by EDX and shown to contain 4.5 at.\% Ga, in both

238 the AZO and GZO films carbon content was below 5 at.\% (approaching detection limit) no chlorine contamination could be detected. 

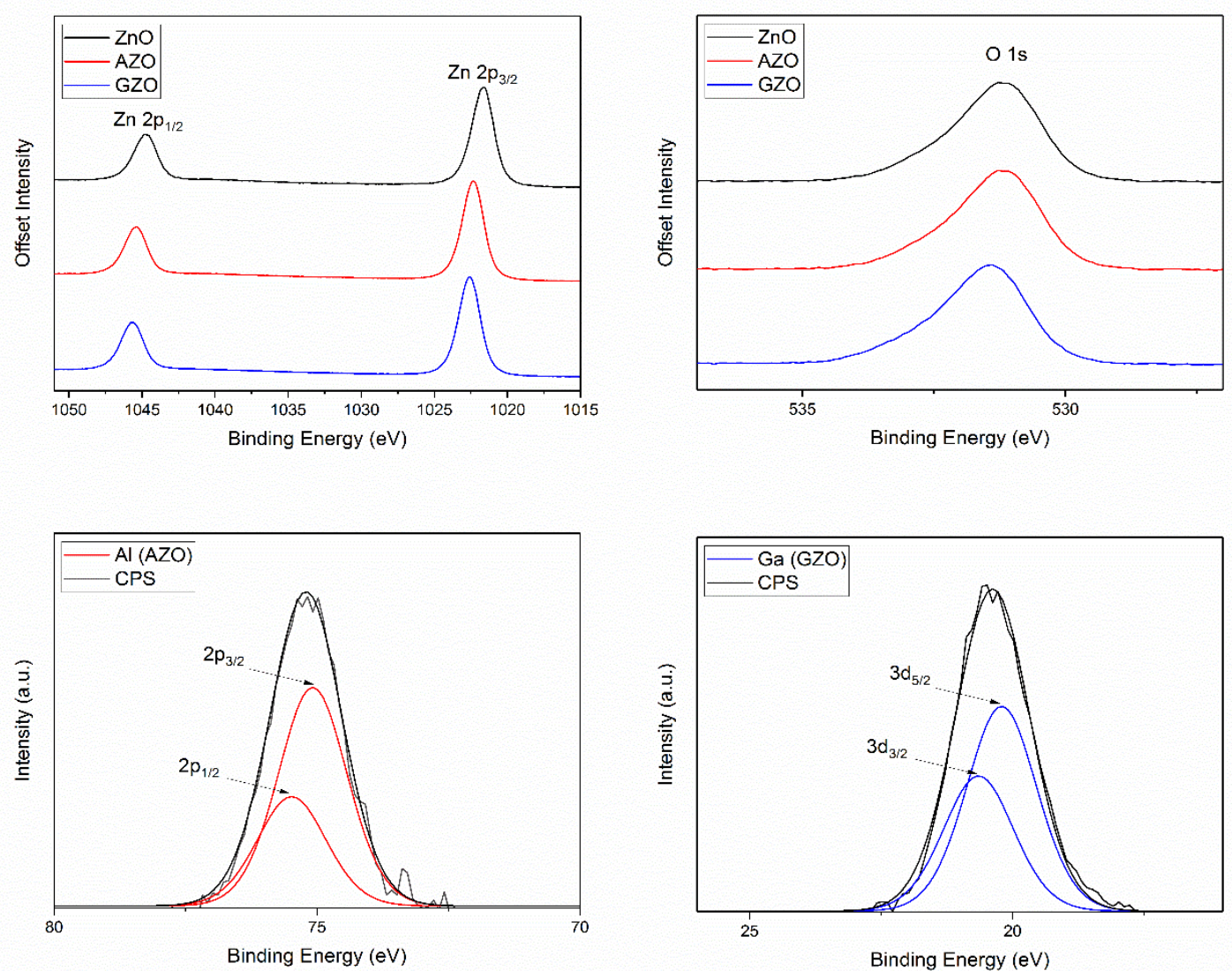

Fig. 2: XPS analysis of: $\mathrm{Zn} 2 \mathrm{p}$ peaks in $\mathrm{ZnO}$ (black), $A Z O$ (red) and GZO (blue) (top left); $O$ 1s peaks in $\mathrm{ZnO}$ (black), AZO (red) and GZO (blue) (top right); Al $2 p$ in AZO (red) (bottom left); and Ga 3d in GZO (blue) 243 (bottom right).

244 In the X-ray photoelectron spectroscopy (XPS) data all peaks were fitted by a Gaussian/Lorentzian product distribution. For films deposited from 1 a Zn:O ratio of 1.1:1.0 was calculated, indicative of binary ZnO with a $\mathrm{Zn} 2 \mathrm{p}_{3 / 2}$ binding energy of $1021.5 \mathrm{eV}$.[28] In Fig. 2 (top left) the $\mathrm{Zn} 2 \mathrm{p}$ peaks are offset for ZnO (black), AZO (red) and GZO (blue), a slight shift, consistent with the presence of $\mathrm{Al}$ and Ga dopants, respectively, was observed for the $\mathrm{Zn} 2 \mathrm{p}_{3 / 2}$ binding energy: $1022.7 \mathrm{eV}$ (AZO) and $1022.3 \mathrm{eV}$ (GZO). The $\mathrm{O} 1 \mathrm{~s}$ spectra recorded for the $\mathrm{ZnO}, \mathrm{AZO}$ and GZO films (Fig. 2, top right) all included a peak located between $530-534 \mathrm{eV}$. This can be assigned to $\mathrm{O}^{2-}$ ions in the $\mathrm{ZnO}$ hexagonal wurtzite structure. The appearance of a shoulder in each of the spectra correspond to the presence of loosely bound surface species.

XPS confirmed the presence of $\mathrm{Al}(\mathrm{III})$ in the AZO films deposited from 1 and $\mathbf{2}$, the binding energy of $\mathrm{Al} 2 \mathrm{p}_{3 / 2}$ was $75.1 \mathrm{eV}$ and the $\mathrm{Al} 2 \mathrm{p}_{1 / 2}$ was $75.5 \mathrm{eV}$ (Fig. 2, bottom left), higher than previous reports for $\mathrm{Al}_{2} \mathrm{O}_{3}$, , 22 ] which is consistent for Al as a dopant as opposed to when incorporated within its binary oxide.[29] In the XPS of the GZO films deposited from 1 and $\mathbf{3}$, the binding energy of the Ga $3 d_{5 / 2}$ at $20.2 \mathrm{eV}$ is indicative of $\mathrm{Ga}$ (III) (Fig. 2, bottom right). Since this technique only probes the surface and not the bulk of the material and this, 

aluminium or gallium could not be calculated from the XPS data.[30]

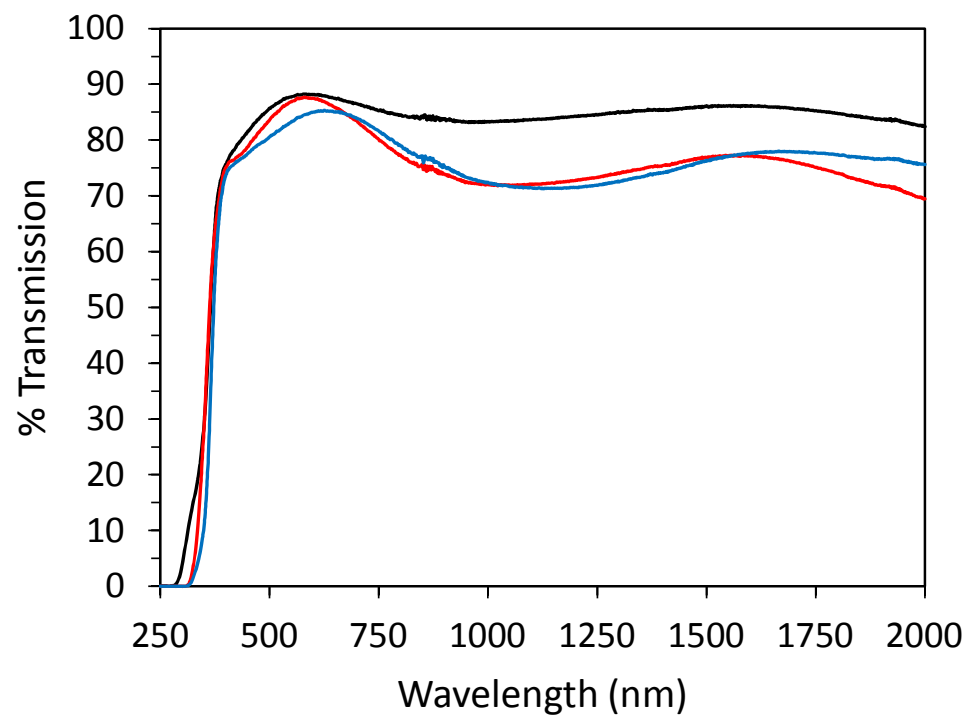

259

Fig. 3: UV/vis data for $\mathrm{ZnO}$ thin film deposited from 1 at $400^{\circ} \mathrm{C}$ (black); AZO film deposited from 1 and 2 at $400^{\circ} \mathrm{C}$ (red); GZO film deposited from 1 and 3 at $400^{\circ} \mathrm{C}$ (blue).

Using UV/Vis spectroscopy, it was possible to obtain information using transmission reflectance data about the transparency and bandgap of the thin films of $\mathrm{ZnO}, \mathrm{AZO}$ and $\mathrm{GZO}$ by using a Tauc plot. A value of $3.13 \mathrm{eV}$ was calculated in accordance with literature values for the band gap of the thin films of ZnO deposited using 1.[9] Comparatively the band gap of the AZO films deposited from compounds $\mathbf{1}$ and $\mathbf{2}$ and the GZO films deposited from 1 and 3 were found to be 3.25 and $3.28 \mathrm{eV}$ respectively. The increase in the bandgap upon doping may be expected due to an increased charge carrier concentration due to the Burnstein-Moss effect widening the bandgap.[14] The slightly wider band gap contributes to the high optical transparency of the films. The data showed transmission exceeded $80 \%$ in all films in the visible region fitting the requirements for a TCO.

Resistance of the films was determined by two-point probe, additionally, using the film thickness obtained from Filmetrics equipment the resistivity was calculated. Table 1 summarises the data for the ZnO, AZO and GZO films.[26]

\section{Table 1}

Summary of the characterisation of $\mathrm{ZnO}, \mathrm{AZO}$ and GZO thin films: film thickness $(\mathrm{d})$, sheet resistance $\left(\mathrm{R}_{\mathrm{Sh}}\right)$, bulk resistivity $(\rho)$, cell parameters were calculated using a Le Bail refinement, position of the (002) peak in the XRD pattern $\left({ }^{\circ}\right)$, band gap $\left(E_{g}\right)$, at.\% of dopant from EDX.

\begin{tabular}{|c|c|c|c|c|c|c|c|}
\hline Film & $\mathrm{d}(\mathrm{nm})$ & $\begin{array}{l}\mathrm{R}_{\mathrm{Sh}} \\
\left(k \Omega \mathrm{sq}^{-1}\right)\end{array}$ & $\rho(\Omega \mathrm{cm})$ & $\begin{array}{l}\text { Cell parameters } \\
(\AA \AA)\end{array}$ & $\begin{array}{l}(002) \\
\text { Peak } \\
\text { position } \\
\left({ }^{\circ}\right)\end{array}$ & $\begin{array}{l}E_{g} \\
(\mathrm{eV})\end{array}$ & $\begin{array}{l}\text { EDX } \\
\text { (at\%) }\end{array}$ \\
\hline
\end{tabular}




\begin{tabular}{lccccccc}
\hline ZnO & 182.6 & 70 & 1.270 & $\begin{array}{l}a=3.2367(5) \\
b=5.1613(5)\end{array}$ & 34.85 & 3.13 & - \\
AZO & 210.3 & 12 & 0.252 & $\begin{array}{l}a=3.2353(5) \\
b=5.1785(5)\end{array}$ & 35.00 & 3.25 & $4.8(\mathrm{Al})$ \\
GZO & 189.1 & 40 & 0.756 & $\begin{array}{l}a=3.2257(5) \\
b=5.1496(5)\end{array}$ & 34.90 & 3.28 & $4.5(\mathrm{Ga})$ \\
\hline
\end{tabular}

277 A lower resistivity was recorded in the doped films compared to the ZnO indicating that the addition of 278 dopant improved the electrical properties significantly (Table 1). However, the effect of film thickness must 279 be noted and although not a direct correlation, thicker films generally had better conductivity.
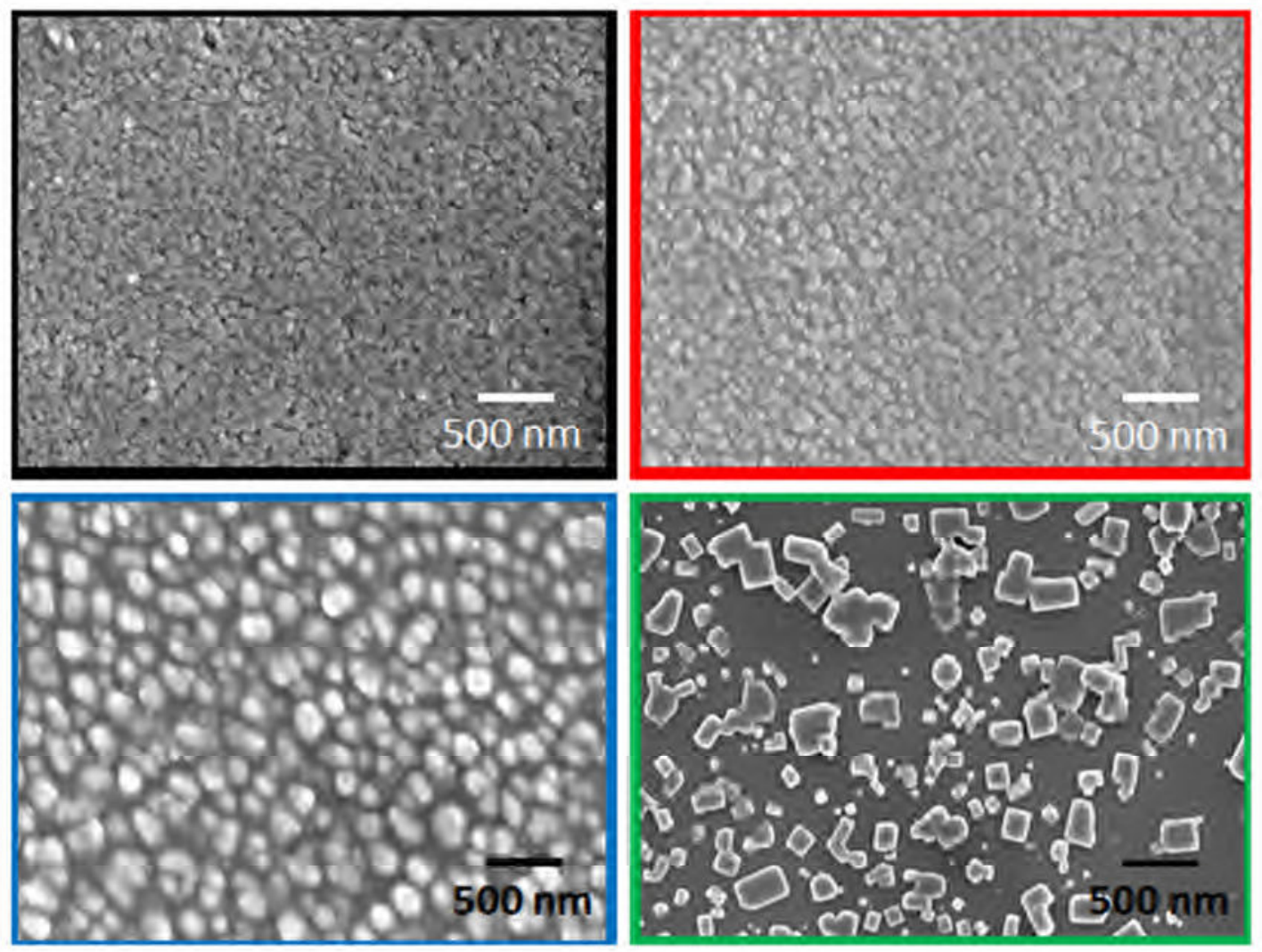

Fig. 4: SEM images of thin films grown at $400^{\circ} \mathrm{C}$ of: $\mathrm{ZnO}$ deposited from 1 (black border); b) AZO deposited from a one-pot mixture of $\mathbf{1}$ and $\mathbf{2}$ (red border); GZO deposited from a one-pot mixture of $\mathbf{1}$ and $\mathbf{3}$ (blue border); and the crystalline spinel: $\mathrm{GaZn}_{2} \mathrm{O}_{4}$ produced from heating $\mathrm{GZO}$ thin films on quartz substrates at $1000^{\circ} \mathrm{C}$ (green border).

Scanning electron microscopy (SEM) shows the surface morphology of the zinc oxide films deposited from 1 (Fig. 4, black border) to be similar in appearance from the AZO films indicating that doping, in this instance seems to have little effect on the morphology. Interestingly however the GZO films appeared to be formed of larger spherical units (Fig. 4, blue border). These GzO films were also deposited on quartz and then annealed at $1000{ }^{\circ} \mathrm{C}$. The relatively equally sized clusters $(\sim 70 \mathrm{~nm})$ observed on the GZO films were replaced with an array of crystalline blocks, after annealing (Fig. 4, green border). 
The Kirkendall effect - namely bulk diffusion at the interface of two metal layers which produces both vacancies and new materials - has been exploited in recent years for the synthesis of hollow nanomaterials. [31,32] A mechanism analogous to this effect has been used to synthesize spinel structures of the type $\mathrm{AB}_{2} \mathrm{O}_{4}$, from a range of techniques including $A L D$ and CVD whereby layers of different materials are heated above a certain temperature.[33-35] It is likely that the GZO films presented herein have an amorphous layer of oxide at their surface. This can be difficult to detect in films deposited at $400{ }^{\circ} \mathrm{C}$ because $\mathrm{Ga}_{2} \mathrm{O}_{3}$ is amorphous at this temperature ruling out XRD analysis, furthermore the low doublet separation in the XPS for gallium implies that any number of environments can fit the single peak recorded. In order to investigate the effect this layer may have on the material beneath, films of GZO were annealed at $1000{ }^{\circ} \mathrm{C}$. The XRD data below (Fig. 5) confirms the spinel zinc gallate, $\mathrm{ZnGa}_{2} \mathrm{O}_{4}$ formed alongside $\mathrm{Ga}_{2} \mathrm{O}_{3}$. Annealing the film had therefore caused the formation of $\mathrm{ZnGa}_{2} \mathrm{O}_{4}$ from a reaction between $\mathrm{Ga}_{2} \mathrm{O}_{3}$ present on

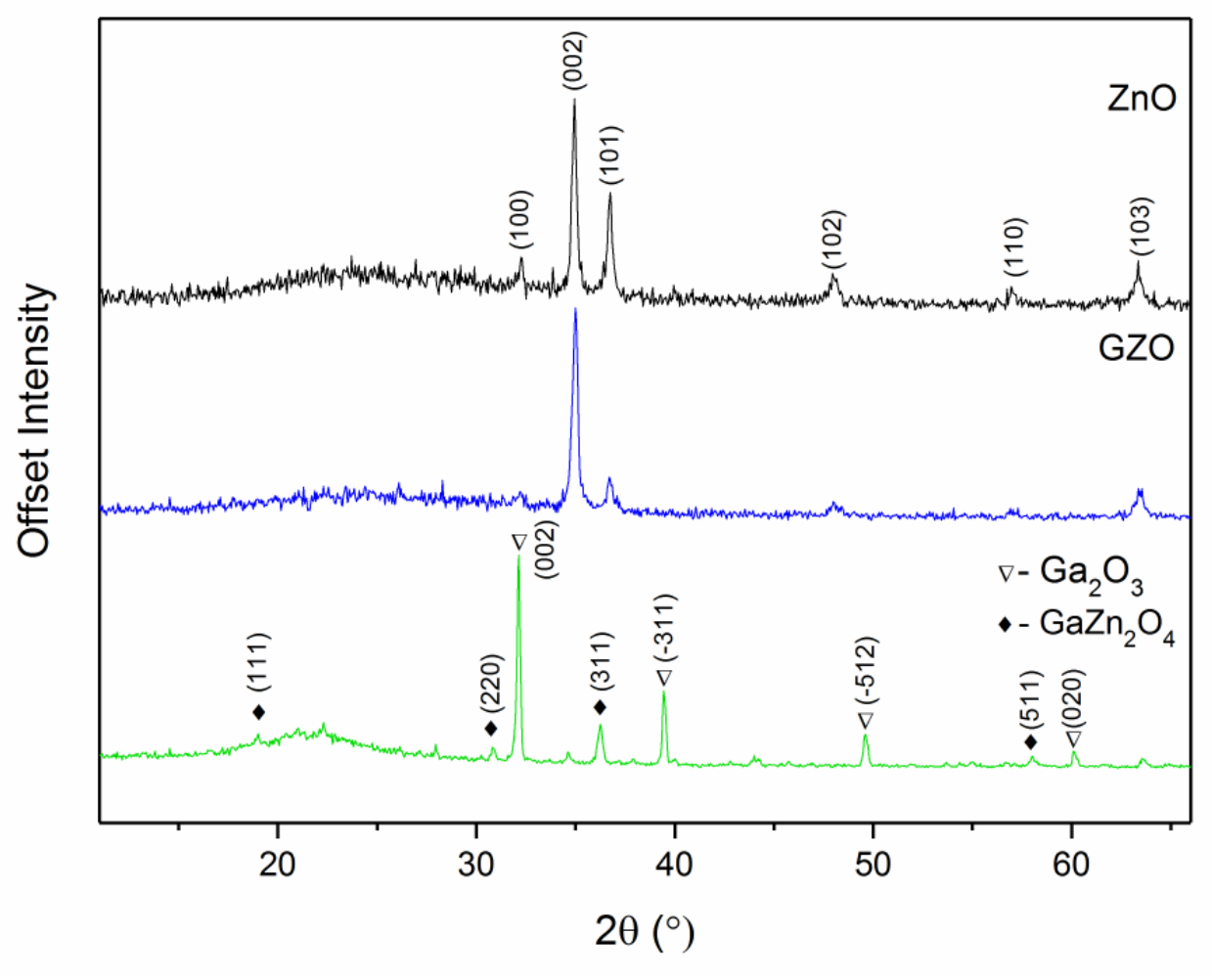

Fig 5: XRD spectra of ZnO on glass (black); GZO film deposited on glass (blue) and GZO on quartz annealed at $1000^{\circ} \mathrm{C}$ (green). Diamond markers in the annealed spectrum show where Bragg peaks occur characteristic of zinc gallate, triangle markers show $\mathrm{Ga}_{2} \mathrm{O}_{3}$.

$\mathrm{Ga}_{2} \mathrm{O}_{3}$ and $\mathrm{GaZn}_{2} \mathrm{O}_{4}$ are the thermodynamically stable products at $1000{ }^{\circ} \mathrm{C}$ when compared to GZO. These results confirm AACVD as a simple alternative to some multistep processes[5] which are currently used to deposit spinels consistent with previous investigations into post-annealing temperatures.[36] 
Following the synthesis of zinc, aluminium and gallium containing compounds using the same $\beta$-ketoimine ligand - each containing pre-formed metal-oxygen bonds, AACVD has been used to deposit in a simple one step procedure thin films of $\mathrm{ZnO}, \mathrm{AZO}$ and GZO. Films were deposited on glass at $400{ }^{\circ} \mathrm{C}$, and in the case of GZO, also on quartz for post-annealing treatment confirming the formation of the thermodynamically favourable spinel $\mathrm{GaZn}_{2} \mathrm{O}_{4}$. Dopant concentration in the films matches the amount added to the reaction pot, proving this to be an efficient and non-wasteful technique. In conclusion the thin films deposited using these precursors showed reasonable electrical conductivity and showed excellent optical properties; a promising proof of concept for one-pot made-to-measure precursor systems.

\section{Acknowledgements}

C. E. K. thanks the Ramsay Memorial Trust for funding. C. J. C thanks the EPSRC for the grant: EP/K001515.

[1]C.E. Knapp, C.J. Carmalt, Solution based CVD of main group materials, Chem Soc Rev. 45 (2016) 1036-1064. doi:10.1039/C5CS00651A.

[2]B.G. Lewis, D.C. Paine, Applications and Processing of Transparent Conducting Oxides, MRS Bull. 25 (2000) 22-27. doi:10.1557/mrs2000.147.

[3]Y.-H. Liu, Y.-C. Cheng, Y.-L. Tung, Y. Chi, Y.-L. Chen, C.-S. Liu, S.-M. Peng, G.-H. Lee, Synthesis and characterization of fluorinated ??-ketoiminate and imino-alcoholate Pd complexes: precursors for palladium chemical vapor deposition, J. Mater. Chem. 13 (2003) 135-142. doi:10.1039/b208535f.

[4]S.-U. Park, J.-H. Koh, Electrical and optical properties of in and $\mathrm{Al}$ doped $\mathrm{ZnO}$ thin film, Electron. Mater. Lett. 9 (2013) 493-496. doi:10.1007/s13391-013-0046-9.

[5]E. Fortunato, L. Raniero, L. Silva, A. Goncalves, A. Pimentel, P. Barquinha, H. Aguas, L. Pereira, G. Goncalves, I. Ferreira, Highly stable transparent and conducting gallium-doped zinc oxide thin films for photovoltaic applications, Sol. Energy Mater. Sol. Cells. 92 (2008) 1605-1610. doi:10.1016/j.solmat.2008.07.009.

[6]S.Y. Li, P. Lin, C.Y. Lee, T.Y. Tseng, C.J. Huang, Effect of Sn dopant on the properties of ZnO nanowires, J. Phys. Appl. Phys. 37 (2004) 2274-2282. doi:10.1088/0022-3727/37/16/009.

[7]A. Hafdallah, F. Yanineb, M.S. Aida, N. Attaf, In doped ZnO thin films, J. Alloys Compd. 509 (2011) 7267-7270. doi:10.1016/j.jallcom.2011.04.058.

[8]V.R. Shinde, T.P. Gujar, C.D. Lokhande, R.S. Mane, S.-H. Han, Mn doped and undoped ZnO films: A comparative structural, optical and electrical properties study, Mater. Chem. Phys. 96 (2006) 326-330. doi:10.1016/j.matchemphys.2005.07.045.

[9]K. Ellmer, Resistivity of polycrystalline zinc oxide films: current status and physical limit, J. Phys. Appl. Phys. 34 (2001) 3097-3108. doi:10.1088/0022-3727/34/21/301.

[10] M.N. Islam, T.B. Ghosh, K.L. Chopra, H.N. Acharya, XPS and X-ray diffraction studies of aluminum-doped zinc oxide transparent conducting films, Thin Solid Films. 280 (1996) 20-25. doi:10.1016/0040-6090(95)08239-5.

[11] D.P. Howard, P. Marchand, L. McCafferty, C.J. Carmalt, I.P. Parkin, J.A. Darr, HighThroughput Continuous Hydrothermal Synthesis of Transparent Conducting Aluminum and Gallium Co-doped Zinc Oxides, ACS Comb. Sci. 19 (2017) 239-245. doi:10.1021/acscombsci.6b00118.

[12] C.M. Muiva, T.S. Sathiaraj, K. Maabong, Effect of doping concentration on the properties of aluminium doped zinc oxide thin films prepared by spray pyrolysis for transparent electrode applications, Ceram. Int. 37 (2011) 555-560. doi:10.1016/j.ceramint.2010.09.042. 
[13] L. Schmidt-Mende, J.L. MacManus-Driscoll, $\mathrm{ZnO}$ - nanostructures, defects, and devices, Mater. Today. 10 (2007) 40-48. doi:10.1016/S1369-7021(07)70078-0.

[14] M.-C. Jun, S.-U. Park, J.-H. Koh, Comparative studies of Al-doped ZnO and Ga-doped ZnO transparent conducting oxide thin films, Nanoscale Res. Lett. 7 (2012) 639. doi:10.1186/1556276X-7-639.

[15] E. Fortunato, D. Ginley, H. Hosono, D.C. Paine, Transparent Conducting Oxides for Photovoltaics, MRS Bull. 32 (2007) 242-247. doi:10.1557/mrs2007.29.

[16] S. Chen, G. Carraro, D. Barreca, R. Binions, Growth and electro-optical properties of Ga-doped $\mathrm{ZnO}$ films prepared by aerosol assisted chemical vapour deposition, Thin Solid Films. 584 (2015) 316-319. doi:10.1016/j.tsf.2014.11.092.

[17] S.D. Ponja, S. Sathasivam, I.P. Parkin, C.J. Carmalt, Transparent conductive aluminium and fluorine co-doped zinc oxide films via aerosol assisted chemical vapour deposition, RSC Adv. 4 (2014) 49723-49728. doi:10.1039/C4RA09997D.

[18] D.B. Potter, D.S. Bhachu, M.J. Powell, J.A. Darr, I.P. Parkin, C.J. Carmalt, Al-, Ga-, and Indoped $\mathrm{ZnO}$ thin films via aerosol assisted CVD for use as transparent conducting oxides: Al-, Ga-, and In-doped ZnO thin films via aerosol assisted CVD, Phys. Status Solidi A. 213 (2016) 1346-1352. doi:10.1002/pssa.201532996.

[19] D.S.Y. Jayathilake, T.A.N. Peiris, J.S. Sagu, D.B. Potter, K.G.U. Wijayantha, C.J. Carmalt, D.J. Southee, Microwave-Assisted Synthesis and Processing of Al-Doped, Ga-Doped, and Al, Ga Codoped $\mathrm{ZnO}$ for the Pursuit of Optimal Conductivity for Transparent Conducting Film Fabrication, ACS Sustain. Chem. Eng. 5 (2017) 4820-4829. doi:10.1021/acssuschemeng.7b00263.

[20] D. Pugh, P. Marchand, I.P. Parkin, C.J. Carmalt, Group $13 \beta$-Ketoiminate Compounds: Gallium Hydride Derivatives As Molecular Precursors to Thin Films of $\mathrm{Ga}_{2} \mathrm{O}_{3}$, Inorg. Chem. 51 (2012) 6385-6395. doi:10.1021/ic3006794.

[21] P. Marchand, C.J. Carmalt, Molecular precursor approach to metal oxide and pnictide thin films, Coord. Chem. Rev. 257 (2013) 3202-3221. doi:10.1016/j.ccr.2013.01.030.

[22] C.E. Knapp, P. Marchand, C. Dyer, I.P. Parkin, C.J. Carmalt, Synthesis and characterisation of novel aluminium and gallium precursors for chemical vapour deposition, New J Chem. 39 (2015) 6585-6592. doi:10.1039/C5NJ00947B.

[23] J.S. Matthews, O.O. Onakoya, T.S. Ouattara, R.J. Butcher, Synthesis and characterization of zinc AP-MOCVD precursors and their utility in the growth of ZnO, Dalton Trans. (2006) 3806. doi:10.1039/b603308c.

[24] J. Holmes, K. Johnson, B. Zhang, H.E. Katz, J.S. Matthews, Metal organic chemical vapor deposition of $\mathrm{ZnO}$ from $\beta$-ketoiminates: MOCVD of $\mathrm{ZnO}$ from $\beta$-ketoiminates, Appl. Organomet. Chem. 26 (2012) 267-272. doi:10.1002/aoc.2850.

[25] Z.R. Khan, Optical and Structural Properties of ZnO Thin Films Fabricated by Sol-Gel Method, Mater. Sci. Appl. 02 (2011) 340-345. doi:10.4236/msa.2011.25044.

[26] S.S. Meysami, A.A. Koós, F. Dillon, N. Grobert, Aerosol-assisted chemical vapour deposition synthesis of multi-wall carbon nanotubes: II. An analytical study, Carbon. 58 (2013) 159-169. doi:10.1016/j.carbon.2013.02.041.

[27] S. Liang, Z.-X. Mei, X.-L. Du, Modulation of electrical and optical properties of gallium-doped ZnO films by radio frequency magnetron sputtering, Chin. Phys. B. 21 (2012) 067306. doi:10.1088/1674-1056/21/6/067306.

[28] H.A. Ali, A.A. Iliadis, A. Von Cresce, P. Kofinas, U. Lee, Properties of self-assembled ZnO nanostructures on $\mathrm{Si}$ and $\mathrm{SiO} / \mathrm{sub}$ 2/ wafers, in: IEEE, 2001: pp. 454-457. doi:10.1109/ISDRS.2001.984543.

[29] J.-L. Yan, Y.-N. Zhao, C. Li, Formation of $\mathrm{ZnGa}_{2} \mathrm{O}_{4}$ films by multilayer deposition and subsequent thermal annealing, Chin. Phys. B. 23 (2014) 048105. doi:10.1088/16741056/23/4/048105.

[30] J. Creighton, Ho, P, Introduction to chemical vapor deposition (CVD), ASM International, Materials Park, OH, 2001.

[31] H.J. Fan, M. Knez, R. Scholz, D. Hesse, K. Nielsch, M. Zacharias, U. Gösele, Influence of Surface Diffusion on the Formation of Hollow Nanostructures Induced by the Kirkendall Effect: The Basic Concept, Nano Lett. 7 (2007) 993-997. doi:10.1021/n1070026p. 
[32] H. Jin fan, M. Knez, R. Scholz, K. Nielsch, E. Pippel, D. Hesse, M. Zacharias, U. Gösele, Monocrystalline spinel nanotube fabrication based on the Kirkendall effect, Nat. Mater. 5 (2006) 627-631. doi:10.1038/nmat1673.

[33] C.E. Knapp, J.A. Manzi, A. Kafizas, I.P. Parkin, C.J. Carmalt, Aerosol-Assisted Chemical Vapour Deposition of Transparent Zinc Gallate Films, ChemPlusChem. 79 (2014) 1024-1029. doi:10.1002/cplu.201402037.

[34] C.E. Knapp, I.D. Prassides, S. Sathasivam, I.P. Parkin, C.J. Carmalt, Aerosol-Assisted Chemical Vapour Deposition of a Copper Gallium Oxide Spinel, ChemPlusChem. 79 (2014) 122-127. doi:10.1002/cplu.201300289.

[35] S. Kumar, G. Sarau, C. Tessarek, M. Göbelt, S. Christiansen, R. Singh, Study of high quality spinel zinc gallate nanowires grown using CVD and ALD techniques, Nanotechnology. 26 (2015) 335603. doi:10.1088/0957-4484/26/33/335603.

[36] C.H. Ahn, S.H. Kim, Y.K. Kim, H.S. Lee, H.K. Cho, Effect of post-annealing temperatures on thin-film transistors with $\mathrm{ZnO} / \mathrm{Al} 2 \mathrm{O} 3$ superlattice channels, Thin Solid Films. 584 (2015) 336340. doi:10.1016/j.tsf.2015.01.017. 